\title{
The New 2020 European Society of Cardiology Guidelines for the Management of Adults with Congenital Heart Disease: A Perspective of the Interamerican Adult Congenital Heart Disease Council of the Interamerican Society of Cardiology (ACHDC-IASC)
}

\author{
John Jairo Araujo MD* iD
}

Department of Clinic and Research in Pediatric and Adult Congenital Heart Disease, Somer Incare Cardiovascular Center, Rionegro, Colombia

\begin{abstract}
Recently, the new European Society of Cardiology guidelines for the management of adult congenital heart disease were published. Due to the coronavirus pandemic, on this occasion, they were presented virtually during the last European Cardiology Congress. The greater survival of adults with congenital heart disease is a result of the latest advances in new interventionist treatments for repairing congenital heart defects, the implementation of new medical management, and, especially, better risk stratification for catheterization and surgical times. We know today that $90 \%$ of adults with simple defects reach 60 years of age, along with $75 \%$ and $40 \%$ of those with moderate and complex defects, respectively. This success results in more and more adults presenting, with increased prevalence in older ages. This has been the main reason for renewing the management guidelines. The objective of the present document is to highlight the most important and updated aspects of the new guidelines. It describes the perspective of the Interamerican Adult Congenital Heart Disease Council of the Interamerican Society of Cardiology regarding their application for Latin America.
\end{abstract}

Keywords

Adult Congenital Heart Disease, Guidelines, Interamerican Society of Cardiology, Congenital Heart Disease Council

\section{Introduction}

The year 2020 will be remembered as a year of great changes for humanity. The COVID-19 pandemic [1,2] has affected all on-site scientific events, and the most recent 2020 European Cardiology Congress is no exception. It was conducted virtually from August 29 to September 1 [3]. There was great expectation for this congress, not only due to its scientific quality and variety of speakers, but also because the results of several research studies on cardiology would be presented, especially several of the most renewed European clinical practice guidelines $[4,5]$. For the adult congenital heart disease (ACHD) specialty, the enormous anticipation of seeing the new European Society of Cardiology guidelines for managing adults with congenital heart disease (ESC ACHD guidelines) could wait no longer [6]. Thus, the virtual modality has been the best ally on this occasion. As with its predecessor published in 2010 [7], this new version has been developed by members of the Task Force for the Management of Adult Congenital Heart Disease of the European Society of Cardiology previously chosen by the ESC. This task force in- cludes a large select group of global experts on several cardiology topics related to congenital heart disease (CHD). Just as in the previous 2010 guidelines, these have been endorsed by the Association for European Paediatric and Congenital Cardiology (AEPC). However, this new 2020 version also includes the endorsement of the International Society for Adult

*Corresponding author: Dr. John Jairo Araujo MD, Cochair Adult Congenital Heart Disease Council in Inter American Society of Cardiology; Department of Clinic and Research in Pediatric and Adult Congenital Heart Disease, Somer Incare Cardiovascular Center, Rionegro, Colombia

Accepted: November 28, 2020

Published online: November 30, 2020

Citation: Araujo JJ (2020) The New 2020 European Society of Cardiology Guidelines for the Management of Adults with Congenital Heart Disease: A Perspective of the Interamerican Adult Congenital Heart Disease Council of the Interamerican Society of Cardiology (ACHDC-IASC). Ann Public Health Reports 4(1):108-114 
Citation: Araujo JJ (2020) The New 2020 European Society of Cardiology Guidelines for the Management of Adults with Congenital Heart Disease: A Perspective of the Interamerican Adult Congenital Heart Disease Council of the Interamerican Society of Cardiology (ACHDC-IASC). Ann Public Health Reports 4(1):108-114

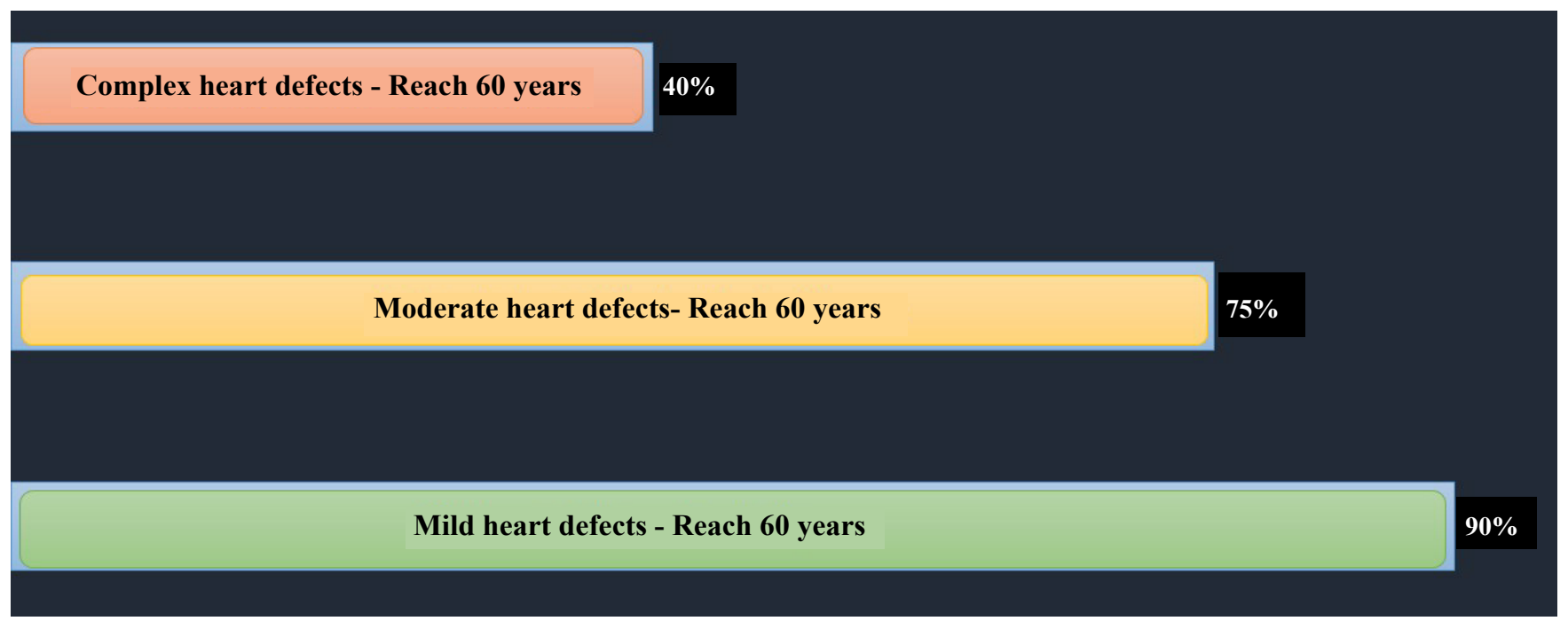

Figure 1: Current survival of adults with congenital heart disease, according to the degree of complexity reaching 60 years.

Congenital Heart Disease (ISACHD). The document is 83 pages long and contains 329 references. It includes several tables and algorithms for making medical, interventionist and surgical decisions in the various CHDs, which makes them very didactic and easy to understand.

\section{Discussion}

\section{Progression and aging of the congenital heart disease population}

Because it is well known that today, in developed countries, there are more adults than children with CHD [8,9]. This has been the tendency in almost all European nations in the last few years. Growth has been noted in the number of increasingly older ACHDs who require more care in the cardiovascular centers. This has been the main reason expressed by the authors for developing the renewed guidelines. As the population ages and more ACHDs survive and reach a greater age, we understand more of the progression and behavior of CHDs. This change is a result of the success in managing ACHDs, and the development of adult CHD units. Advances in new interventionist treatments for repairing $\mathrm{CHDs}$, the implementation of new medical management, and, especially, better risk stratification for catheterization and surgical times $[10,11]$. With greater survival, geriatric syndromes and cognitive and motor disorders, among others, appear early on, increasing the morbidity of CHDs. According to the degree of complexity, today we know that survival to 60 years is $90 \%$, $75 \%$ and $40 \%$ for mild, moderate and complex heart defects, respectively [12] (Figure 1).

The number of ACHDs in Latin America is extrapolated according to the ratio of 3,000 ACHDs per million inhabitants [13]. As has occurred in Europe and North America, the number has been increasing over the last few years. For 2020, more than 1.8 million are estimated for South America, with an estimated annual growth of 5-6\%, which may reach an additional $11 \%$ by 2030 , thus projecting close to 2.2 million ACHDs (Figure 2).

\section{Adult congenital heart disease (ACHD) is a bet- ter term than grown-up congenital heart disease (GUCH)}

The 2010 guidelines, "ESC Guidelines for the Management of Grown-Up Congenital Heart Disease," are now called "2020 ESC Guidelines for the Management of Adult Congenital Heart Disease." This new version of the guidelines has adapted to the global literature in referring to adult CHDs, and the influence of ISACHD can be seen.

The term "GUCH", an acronym for "Grown Up Congenital Heart," was created by Dr. Jane Somerville (British cardiologist, Imperial College London) to refer to ACHDs [14]. It has been very popular and has been used in almost all the European documents having to do with ACHDs $[15,16]$. However, it was not popular in America and the rest of the continents. As previously explained, since ACHDs are more numerous today, with a growing prevalence in older age groups, including the elderly, the term GUCH was not considered to be appropriate, and has been changed to "adults with congenital heart disease (ACHDs)" in the whole document, in order to adapt and concur with the international literature. The ACHDC-IASC is very much in agreement with this point, since the term GUCH is not very appropriate for Spanish texts related to ACHDs, and, although some South American countries have adopted it, or have been influenced by the European literature, it is not popular in Latin America.

\section{Regarding the levels of specialized care and the complexity of congenital heart disease}

There are no changes for these recommendations compared to the 2010 guidelines. The recommendations for ACHD care continue to be, in order:

I. Care only in ACHD centers

II. Shared care between general cardiology and periodic visits to the ACHD center 
Citation: Araujo JJ (2020) The New 2020 European Society of Cardiology Guidelines for the Management of Adults with Congenital Heart Disease: A Perspective of the Interamerican Adult Congenital Heart Disease Council of the Interamerican Society of Cardiology (ACHDC-IASC). Ann Public Health Reports 4(1):108-114

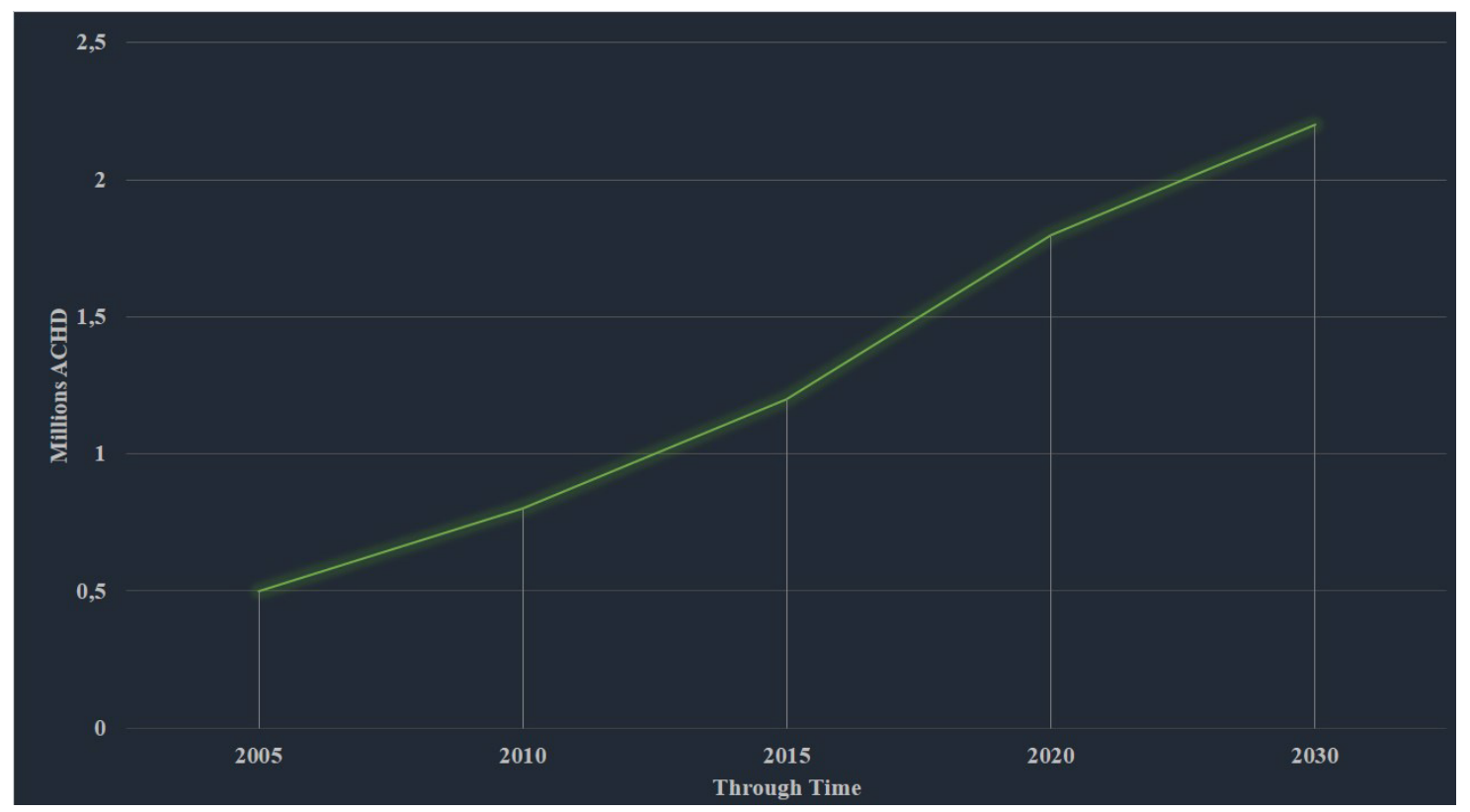

Figure 2: Adults with congenital heart disease in Latin America projected through time.

\section{Level of care only in general cardiology centers}

This somewhat simplified care model tries to provide care coverage for ACHDs, so that all are seen by cardiologists, according to the degree of complexity of the CHD.

The 2001 Bethesda classification established CHD complexity (simple, medium and high) in adults, both for repaired as well as unrepaired CHDs [17]. Up until a few years ago, the experts recommended that adults classified in the simple category could be managed by general cardiologists, adults in the moderate category could be managed together with an ACHD cardiologist, and high complexity adults could only be managed by ACHD cardiologists. However, this concept is now questioned; over time, as the population ages and more adults with CHD reach the fifth or sixth decade of life, more is known about the natural history of many CHDs, especially the complex ones [18].

The Bethesda classification has been purely morphological or anatomical, and does not take the patient's functional status into account. This point is weak, compared to the 2018 AHA/ACC - ACHD guidelines, in which a very strong point was the inclusion of the anatomic-physiological (AP) class, which integrates the anatomical or morphological part of the repaired or unrepaired CHD with the New York Heart Association (NYHA) functional class and nine clinical variables. This establishes four physiological stages: A, B, C and D, in order from lower to greater severity, respectively [19].

This AP classification is becoming increasingly important and has already been validated in recent studies as a predictor of 15-year mortality [20]. The ACHDC-IASC, in previously published documents, has expressed the importance of applying the AP classification to all ACHDs [21,22], and encourages the application of clinical practice guidelines in ACHDs, all within the context of the Latin American population.

A point to highlight in the current guidelines is the inclu- sion of pulmonary vascular disease, both in repaired and unrepaired $\mathrm{CHD}$, within the category of complex $\mathrm{CHD}$. This new topic is especially important in univentricular heart cases, where the new treatments with pulmonary vasodilators are gaining importance in the treatment of this group of patients $[23,24]$.

\section{Congenital heart disease is a chronic, life-long condition}

This is the first time that official ACHD guidelines draw a picture alluding to $\mathrm{CHD}$ as a chronic, life-long condition. The central illustration shows the various stages of life and the specific care needs. Congenital heart diseases are a condition which is expressed even before birth. Various heart defects have clinical expression in the fetal stages, manifesting as arrhythmias, heart failure, valve abnormalities, and alterations of the heart chambers (for example, severe Ebstein's anomaly). This affects the fetus in various ways, such as in failure to thrive, hydrops or death, among others [25], showing the phenotypic aggressiveness of many CHDs. After the fetal stage, the neonatal presentation of CHDs may be very diverse. Depending on the severity, some may be undetected and have relapses days, weeks or months after birth.

For American countries, CHDs have a global prevalence of 8-13 cases per 1,000 live births, with close to $45 \%$ being of medium and high complexity, and at least $25 \%$ needing surgical intervention within the first year of life, due to the severity of the defect [26]. These procedures may be repetitive throughout childhood, with multiple hospitalizations and relapses. After the childhood stage, there may be frequent surgical reinterventions and relapses in adolescence.

Finally, in adulthood, all the accumulation or burden of CHD will be expressed in not only cardiovascular disorders, but also systemic effects involving target organs (kidney, liver, brain, heart) and altered homeostasis of several systems (im- 
Citation: Araujo JJ (2020) The New 2020 European Society of Cardiology Guidelines for the Management of Adults with Congenital Heart Disease: A Perspective of the Interamerican Adult Congenital Heart Disease Council of the Interamerican Society of Cardiology (ACHDC-IASC). Ann Public Health Reports 4(1):108-114

munological, hematopoietic, endocrine, gastrointestinal, and musculoskeletal, among others), behaving as a multisystemic disease.

This highly varied phenotypic expression of the CHDs is what is known as CHD heterogeneity, and has been widely discussed and explained in documents previously published by ACHDC-IASC [27]. The widely variable clinical presentation of the same CHD in adults has taught us that not all cases should be approached and managed in the same way. The appearance of concomitant diseases in adults may add severity to the clinical expression of the $\mathrm{CHD}$. The progressive changes and decomposition manifest as heart failure (HF), arrhythmias, syncope, hemorrhages, pulmonary arterial hypertension, endocarditis, pulmonary thromboembolism and stroke, among others [28].

The message previously expressed by ACHDC-IASC is that CHDs are really a heterogenous disease which require heterogeneity of care, with participation of parallel specialties and non-cardiac specialties [29]. Congenital heart disease patients should never be discharged from cardiology; they are and will be a life-long condition [30].

Care should involve not only the ACHD specialist, but also other non-cardiovascular adult specialties such as: Nephrology, pulmonology, neurology, hematology, endocrinology, high-risk obstetrics and gynecology, cardiac pathology, psychology, psychiatry, social work, genetics, gastroenterology, and infectious disease, among others. Other specialties should also actively participate, such as: Hemodynamics, electrophysiology, cardiac rehabilitation, heart failure and intensive care. Finally, other specialties parallel to cardiology should participate, such as cardiovascular diagnostic and interventionist radiology and cardiovascular anesthesiology [19].

A strength of the recent guidelines is that they emphasize the organization of work groups to manage each special situation in ACHDs, and ACHDC-IASC strongly agrees with this idea.

\section{Classes of recommendation and levels of evi- dence}

-Class I: Always recommended

-Class II: Levels of controversy

Ila: Should be considered

Ilb: Could be considered

III: Not recommended

Levels of evidence: A, B, C

This is practically the same as the 2010 guidelines, and there are no changes. Their implementation is applied in all aspects related to decision making and actions. As for therapeutic, interventionist and surgical actions, these are duly highlighted in the sections on each specific CHD.

\section{Main new topics developed}

Arrhythmias: It is well known that one of the main consequences of $\mathrm{CHD}$ relapses is arrhythmias. They present in more than $80 \%$ of $A C H D$ s and are the main reason for consulting [31]. The recent guidelines highlight the importance of knowing their mechanism and emphasize the importance of understanding the structural cause and the anatomy of the underlying CHD. This new version underscores the importance of a multidisciplinary approach for optimal treatment of arrhythmias before, or concomitantly with, percutaneous treatment or surgical interventions. And they highlight the importance of early ablation. The design of a table of risk estimates for arrhythmic events and bradycardias in ACHDs stands out, as well as the risk stratification for sudden cardiac death.

Eisenmenger syndrome/pulmonary arterial hypertension: Without a doubt, this is an extremely important point for ACHDs. At least $10 \%$ of ACHDs are known to develop pulmonary hypertension (PAH), and of these, $50 \%$ develop Eisenmenger syndrome [32-34]. This new version emphasizes risk assessment in all patients with PAH and CHD. It establishes treatment guidelines with monotherapy or combination therapy, according to the case. It determines the indications and recommendations with their respective levels of evidence for when heart defects should or should not be closed. Emphasizing that those with more than five Wood units are considered to be category III.

Univentricular heart and Fontan: The recent guidelines establish some therapeutic recommendations in Fontan circulation. Indications for the use of vasodilators, indications for invasive hemodynamic studies and the electrophysiological approach to arrhythmias.

Cyanotic congenital heart disease: This group of CHDs are known to be high risk due to the various associated complications. The new guidelines stratify risk and intervention activities to decrease the impact. They briefly describe the consequences of chronic hypoxia and the approach for proper management.

On this point, the ACHDC-IASC's publications have very clearly stated the guidelines for the best treatment path, so as to not harm and worsen the patients' hemodynamic decompensation [35]. This is very important for Latin American countries, as the ACHDC-IASC has analyzed the ACHD population profile in Latin America. Based on previous publications [29], we now know that at least $30 \%$ of ACHDs are newly diagnosed; they are adults who were never diagnosed as having CHD and, therefore, the clinical expression is more severe, due to lack of treatment and monitoring. Likewise, of those who were diagnosed in childhood or adolescence, up to $30 \%$ abandoned treatment and are now adults with incomplete $\mathrm{CHD}$ repair, and thus a more severe clinical phenotype (Figure 3).

Concluding that, in regard to CHDs in Latin American adults, we must be prepared for a sicker population compared to developed countries, where newly diagnosed ACHDs account for less than $10 \%$ of the cases [36].

Biomarkers: Importance is given to neurohormone and myocardial injury (high-sensitivity troponins) or inflammatory (high-sensitivity C-reactive protein) marker measurements. Among the neurohormones, natriuretic peptides [type $B$ 
Citation: Araujo JJ (2020) The New 2020 European Society of Cardiology Guidelines for the Management of Adults with Congenital Heart Disease: A Perspective of the Interamerican Adult Congenital Heart Disease Council of the Interamerican Society of Cardiology (ACHDC-IASC). Ann Public Health Reports 4(1):108-114

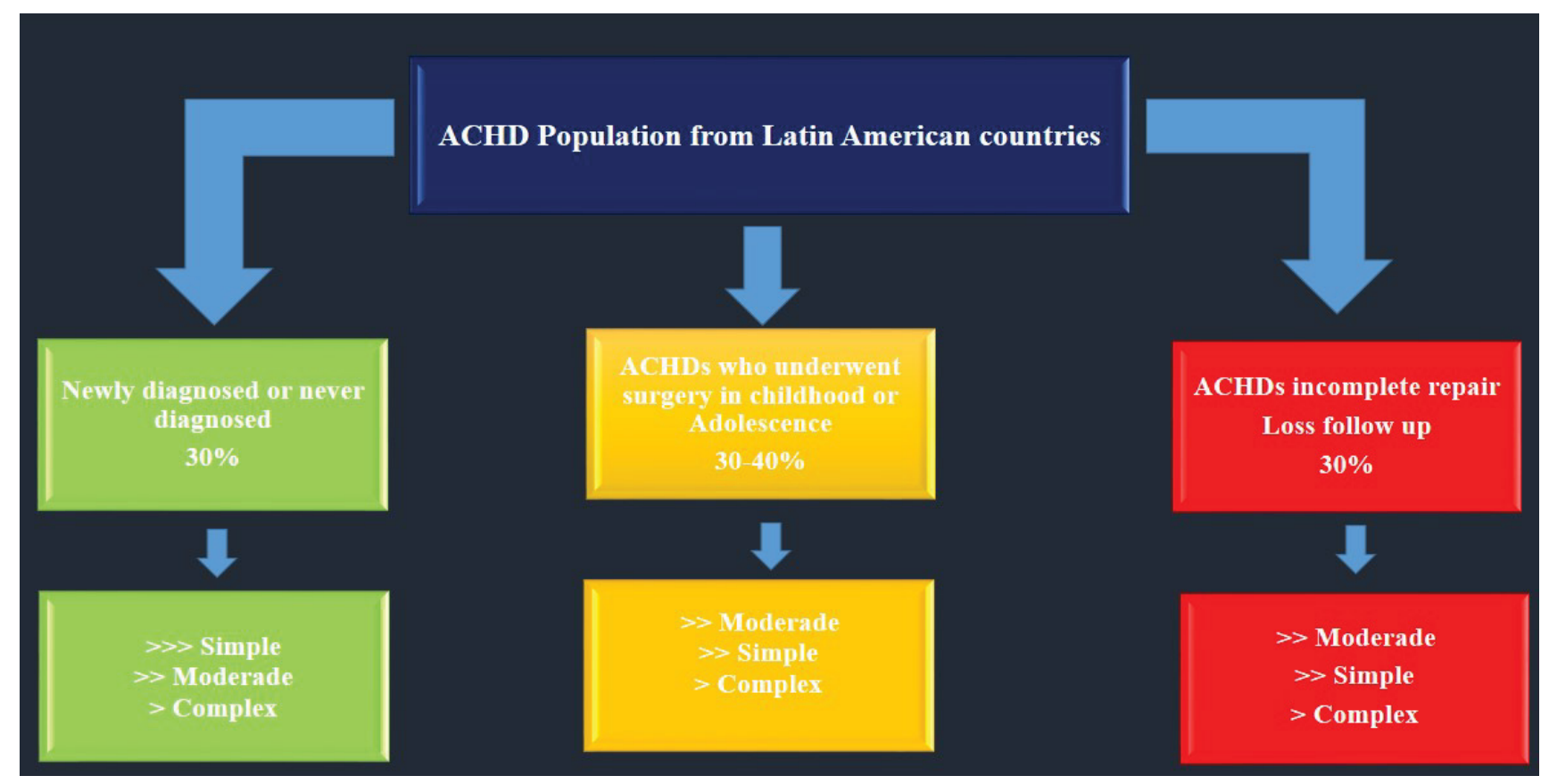

Figure 3: Current profile of the Adult population with congenital heart disease from Latin American countries. Frecuency order: >>> More frequent, >> Medium frequent, > Low frequent.

natriuretic peptide (BNP) and N-terminal pro-BNP (NT-pro$\mathrm{BNP})$ ] are the best and most used in biventricular hearts, but are less sensitive in univentricular and Fontan hearts. In general, they play a role in identifying patients at risk of adverse effects and are prognostic markers. It is emphasized that, in cyanotic CHDs, the levels are elevated due to hypoxia and are harder to interpret, with as yet unstandardized cut-off points.

Heart failure: Heart failure (HF) is a common problem, affecting $20-50 \%$ of the ACHD population, and is the main cause of death. The incidence is increasing and is probably underestimated. The signs and symptoms of HF may occur frequently. Patients at high risk of developing HF require systematic follow-up and diagnostic screening. The ACHDC-IASC has given importance to $\mathrm{HF}$, high lighting that $\mathrm{CHD} H F$ has a multifactorial etiology $[37,38]$.

As with the previous, 2010, guidelines, there is still little evidence on the effective treatment of HF in ACHDs. For now, most of the therapeutic actions are extrapolated from the management of adults without CHD.

Use of anticoagulants: Several comments are made on the risks and benefits of anticoagulation, especially the use of vitamin $\mathrm{K}$ antagonists in cyanotic $\mathrm{CHD}$ patients with $\mathrm{PAH}$. In general, these medications are not recommended in this group of CHDs. There is still insufficient evidence of their benefit. The CHA2DS2-VASc and HAS-BLED scores, used in adults with non-congenital heart disease, are not valid in ACHDs and their use is not recommended.

End-of-life treatment or care: Importance is given to the CHD patients' right to know about their future and survival prognosis. Regardless of their CHD, many patients are concerned about their survival prognosis. These guidelines encourage the participation of patients and their families in the decisions that must be made in terminal cases. Provide palliative care and support options at the end of life.

There are new recommendations regarding right ventricular outflow tract obstruction, tetralogy of Fallot, Ebstein's anomaly, coronary abnormalities and aortopathies.

Finally, there is a new section on quality indicators which aims to validate the applicability of the clinical practice guidelines, to measure and improve adherence. This point is very important, as it has not been done before, and the $2018 \mathrm{AHA} /$ ACC - ACHD guidelines do not have a topic like this, either.

\section{Conclusions and application for Latin America}

The ACHDC-IASC fosters the use and application of clinical practice guidelines for the care of ACHDs. We highlight that the guidelines are templates, not strict directives. Each case must be assessed on its own, using proper clinical judgment, always encouraging interdisciplinary teamwork. The new ESC ACHD guidelines arrive at a good time of changes in the ACHD population. Although it has been previously explained that the clinical profile of the ACHD population in Latin America is more severe than in developed countries, these guidelines provide very useful information to be adapted and applied in patient treatment, in accordance with the Latin American reality. The new topics developed, along with the numerous treatment algorithms, supply information that helps make key and far-reaching decisions, especially for surgery. Ever since the creation of ACHDC-IASC in 2018, it has worked hard to integrate the American cardiologists responsible for the care of ACHDs, with the enthusiasm of an ever-increasing number of members. The time has come for the inter-American community to begin constructing its own ACHD treatment guidelines, and this will be one of the council's objectives. 
Citation: Araujo JJ (2020) The New 2020 European Society of Cardiology Guidelines for the Management of Adults with Congenital Heart Disease: A Perspective of the Interamerican Adult Congenital Heart Disease Council of the Interamerican Society of Cardiology (ACHDC-IASC). Ann Public Health Reports 4(1):108-114

\section{Acknowledgements}

To D. Lynn and Blanquita, Thanks for your support.

\section{Conflicts of Interests}

I have no conflicts of interest to declare.

\section{Funding}

None.

\section{References}

1. World Health Organization (2019) Novel Coronavirus - Situation report. COVID 19 Pandemia.

2. Huang C, Wang Y, Li X, et al. (2020) Clinical features of patients infected with 2019 novel coronavirus in Wuhan, China. The Lancet 395: 497-506.

3. ESC (2020) ESC Congress 2020 - The Digital Experience.

4. Collet J, Thiele H, Barbato E, et al. (2020) 2020 ESC Guidelines for the management of acutecoronary syndromes in patients presenting with out persistent ST-segment elevation: The task force for the management of acutecoronary syndromes in patients presenting with out persistent ST-segmentelevation of the European Society of Cardiology (ESC). European Heart Journal 1-79.

5. Hindricks G, Potpara T, Dagres N, et al. (2020) 2020 ESC Guidelines for the diagnosis and management of atrial fibrillation developed in collaboration with the European Association of Cardio-Thoracic Surgery (EACTS). European Heart Journal 1-125.

6. Baumgartner H, Backer J, Babu-Narayan S, et al. (2020) 2020 ESC Guidelines for the management of adult congenital heart disease: The task force for the management of adult congenital heart disease of the European Society of Cardiology (ESC). European Heart Journal 1-83.

7. Baumgartner H, Bonhoeffer P, De Groot N, et al. (2010) ESC Guidelines for the management of grown-up congenital heart disease (new version 2010). Eur Heart J 31: 2915-2957.

8. Baumgartner $\mathrm{H}$ (2014) Geriatric congenital heart disease: A new challenge in the care of adults with congenital heart disease? Eur Heart J 35: 683-685.

9. Gilboa S, Devine O, Kucik J, et al. (2016) Congenital heart defects in the United States estimating the magnitude of the affected population in 2010. Circulation 134: 101-109.

10. Brida M, Gatzoulis M (2019) Adult congenital heart disease: Past, present and future. Acta Paediatr 108: 1757-1764.

11. Diller G, Kempny A, Babu-Narayan S, et al. (2019) Machine learning algorithms estimating prognosis and guiding therapy in adult congenital heart disease: Data from a single tertiary centre including 10019 patients. Eur Heart J 40: 1069-1077.

12. Van der Bom T, Mulder B, Meijboom F, et al. (2015) Contemporary survival of adults with congenital heart disease. Heart 101: 1989 -1995.

13. Araujo J (2020) Adults with congenital heart disease in the americas - where we are today and where we are heading: A general view of the inter-american adult congenital heart disease council. Journal of Integrative Cardiology Open Access 3: 2-5.

14. Somerville J (1997) Management of adults with congenital heart disease: An increasing problem. Annu Rev Med 48: 283-293.

15. Baumgartner H, Budts W, Chessa M, et al. (2014) Recommen- dations for organization of care for adults with congenital heart disease and for training in thesubspecialty of 'grown-up congenital heart disease' in Europe: A position paper of the working groupon grown-up congenital heart disease of the european society of cardiology. EurHeart J 35: 686-690.

16. Somerville J (2002) Grown-up congenital heart (GUCH) disease: Current needs and provision of service for adolescents and adults with congenital heart disease in the UK. Heart 88: 1-14.

17. Weeb G, Williams R (2001) 32nd Bethesdaconference: Care of the adult with congenital heart disease. J Am Coll Cardiol 37: 1161-1198.

18. Araujo JJ (2019) Units for transitioning pediatric cardiology to adult care with congenital heart disease: Why, when and how? J Cardiol Catheter 37-45.

19. Stout K, Daniels C, Aboulhosn J, et al. (2019) 2018 AHA/ACC Guideline for the management of adults with congenital heart disease: Executive summary: A report of the American College of Cardiology/American Heart Association Task forcé on clinical practice guidelines. J Am Coll Cardiol 73: 1494-1563.

20. Ombelet F, Goossens E, Van De Bruaene A, et al. (2020) Newly developed adult congenital heart disease anatomic and physiological classification: First predictive validity evaluation. J Am Heart Assoc 9: e014988.

21. Araujo J (2018) Commentary on the New 2018 AHA/ACC Guideline for the management of adults with congenital heart disease. CPQ Cardiology 1: 1-10.

22. (2020) Prediction of sudden cardiac death in adults with congenital heart disease. Inter-American Society of Cardiology.

23. Hebert A, Mikkelsen U, Thilen U, et al. (2014) Bosentan improves exercise capacity in adolescents and adult safter Fontan operation: The TEMPO (Treatment With Endothelin Receptor Antagonist in FontanPatients, a Randomized, Placebo-Controlled, Double-Blind Study Measuring Peak Oxygen Consumption) study. Circulation 130: 2021-2030.

24. Wang W, Hu X, Liao W, et al. (2019) The efficacy and safety of pulmonary vasodilators in patients with fontan circulation: $A$ meta-analysis of randomized controlled trials. Pulmonary Circulation 9: 1-9.

25. Paranon S, Acar P (2008) Ebstein'sanomaly of thetricuspid valve: From fetus to adult. Heart 94: 237-243.

26. Liu Y, Chen S, Zühlke L, et al (2019) Global birth prevalence of congenital heart defects 1970-2017: Updated systematic review and meta-analysis of 260 studies. Int J Epidemiol 48: 455-463.

27. (2018) New guidelines for the management of adults with congenital heart disease AHA / ACC-2018. Inter-American Society of Cardiology.

28. Gaeta S, Ward C, Krasuski R (2016) Extra-cardiac manifestations of adult congenital heart disease. Trends in Cardiovascular Medicine 26: 627-636.

29. Araujo J (2019) Adult congenital heart diseaseis really a heterogenous specialty: Message from the colombian adult congenital heart disease chapter. CPQ Cardiology 1: 1-11.

30. Araujo JJ (2018) Adults with congenital heart disease: A growing public health problem? Arch Cardiol Mex 88: 251-252.

31. Hernández A, Paul T, Abrams D, et al. (2018) Arrhythmias in congenital heart disease: A position paper of the European Heart Rhythm Association (EHRA), Association for European Paediatric 
Citation: Araujo JJ (2020) The New 2020 European Society of Cardiology Guidelines for the Management of Adults with Congenital Heart Disease: A Perspective of the Interamerican Adult Congenital Heart Disease Council of the Interamerican Society of Cardiology (ACHDC-IASC). Ann Public Health Reports 4(1):108-114

and Congenital Cardiology (AEPC), and the European Society of Cardiology (ESC) Working Group on Grown-up Congenital heart disease, endorsed by HRS, PACES, APHRS, and SOLAECE. Europace. 20: 1719-1753.

32. Van Riel A, Schuuring M, van Hessen I, et al. (2014) Contemporary prevalence of pulmonary arterial hypertension in adult congenital heart disease following the updated clinical classification. International Journal of Cardiology 174: 299-305.

33. Condliffe R, Clift P, Dimopoulos K (2018) Management dilemmas in pulmonary arterial hypertension associated with congenital heart disease. Pulmonary Circulation 8: 1-12.

34. Kaemmerer H, Apitz C, Brockmeier K, et al. (2018) Pulmonary hypertension in adults with congenital heart disease: Updated recommendations from the cologne consensus conference. Int J Cardiol 272S: 79-88.
35. Araujo JJ (2018) The profile of an adult with congenital heart disease. Int J Clin Cardiol 5: 131.

36. Gatzoulis M, Swan L, Therrien J, et al. (2005) Epidemiology of congenital heart disease. In: Gatzoulis M, Swan L, Therrien J, Pantely G, Adult congenital heart disease: A practical guide. (1st edn), BMJ Books, London, UK, 3-7.

37. Stout K, Broberg C, Book W, et al. (2016) Chronic heart failure in congenital heart disease: A scientific statement from the American heart association. Circulation 133: 770-801.

38. Crossland D, Van De Bruaene A, Silversides C, et al. (2019) Heart failure in adult congenital heart disease: From advanced therapies to end-of-lifecare. Can J Cardiol 35: 1723-1739. 